\title{
CMSSM With Generalized Yukawa Quasi-Unification: An Update
}

\section{N. Karagiannakis}

School of Electrical and Computer Engineering, Faculty of Engineering, Aristotle University of Thessaloniki, Thessaloniki 54124, GREECE

E-mail: nikardauth.or

\section{G. Lazarides}

School of Electrical and Computer Engineering, Faculty of Engineering,

Aristotle University of Thessaloniki, Thessaloniki 54124, GREECE

E-mail: Lazaridedeng.auth.gr

\section{Pallis*}

Departament de Física Teòrica and IFIC,

Universitat de València-CSIC, E-46100 Burjassot, SPAIN

E-mail: cpallisdific.uv.es

\begin{abstract}
We analyze the parametric space of the constrained minimal supersymmetric standard model (CMSSM) with $\mu>0$ supplemented by a generalized asymptotic Yukawa coupling quasi-unification condition which yields acceptable masses for the fermions of the third family. We impose constraints from the cold dark matter abundance in the universe and its direct detection experiments, the $B$ physics, as well as the masses of the sparticles and the lightest neutral CP-even Higgs boson, $m_{h}$. We identify two distinct allowed regions with $M_{1 / 2}>m_{0}$ and $m_{0} \gg M_{1 / 2}$ classified in the hyperbolic branch of the radiative electroweak symmetry breaking. In the first region we obtain, approximately, $44 \lesssim \tan \beta \lesssim 52,-3 \lesssim A_{0} / M_{1 / 2} \lesssim 0.1,122 \lesssim m_{h} / \mathrm{GeV} \lesssim 127$, and mass of the lightest sparticle in the range $(0.75-1.43) \mathrm{TeV}$. Such heavy lightest sparticle masses can become consistent with the cold dark matter requirement on the lightest sparticle relic density thanks to neutralino-stau coannihilations. In the latter region, fixing $m_{h}$ to its central value from the LHC, we find a wider allowed parameter space with milder electroweak-symmetry-breaking fine-tuning, $40 \lesssim \tan \beta \lesssim 50$, $-11 \lesssim A_{0} / M_{1 / 2} \lesssim 15$ and mass of the lightest sparticle in the range $(0.09-1.1) \mathrm{TeV}$. This sparticle is possibly detectable by the present cold dark matter direct search experiments.
\end{abstract}

Proceedings of the Corfu Summer Institute 2014

3-21 September 2014

Corfu, Greece

${ }^{*}$ Speaker. 


\section{Introduction}

One of the most economical and predictive versions of the minimal supersymmetric standard model (MSSM) is the well-known - see e.g Ref. [1] - constrained MSSM (CMSSM) which employs just four and one half free parameters:

$$
\operatorname{sign} \mu, \tan \beta, M_{1 / 2}, m_{0}, \text { and } A_{0},
$$

where sign $\mu$ is the sign of $\mu$, the mass parameter mixing the electroweak Higgs superfields $H_{2}$ and $H_{1}$ of the MSSM which couple to the up- and down-type quarks respectively, $\tan \beta$ is the ratio of the vacuum expectation values of $H_{2}$ and $H_{1}$, while the remaining symbols denote the common gaugino mass, scalar mass, and trilinear scalar coupling, respectively, defined at the grand unified theory (GUT) scale $M_{\mathrm{GUT}}$, which is determined by the unification of the gauge coupling constants.

CMSSM can be further restricted by being embedded in a supersymmetric (SUSY) GUT with a gauge group containing $S U(4)_{\mathrm{c}}$ and $S U(2)_{R}$. This can lead to 'asymptotic' Yukawa unification (YU) [2], i.e. the exact unification of the third generation Yukawa coupling constants at the SUSY GUT scale $M_{\mathrm{GUT}}$. Given the experimental values of the top-quark and tau-lepton masses, the CMSSM supplemented by the assumption of YU (which naturally restricts $\tan \beta \sim 50$ ) yields unacceptable values [3] of the $b$-quark mass for both signs of the MSSM parameter $\mu$. In Ref. [4] - see also Refs. [5-7] -, this problem is addressed in the context of the Pati-Salam (PS) GUT model, without the need of invoking departure from the CMSSM universality. We prefer to sacrifice the exact YU in favor of the universality hypothesis, since we consider the latter as more economical, predictive, and easily accommodated within conventional SUSY GUT models. In particular, the Higgs sector of the simplest PS model [8] is extended so that $H_{2}$ and $H_{1}$ are not exclusively contained in a $S U(4)_{c}$ singlet, $S U(2)_{\mathrm{L}} \times S U(2)_{\mathrm{R}}$ bidoublet superfield, but receive subdominant contributions from another bidoublet too which belongs to the 15 representation of $S U(4)_{c}$. As a result, YU is naturally violated and replaced by a set of asymptotic Yukawa quasi-unification conditions (YQUCs):

$$
h_{t}\left(M_{\mathrm{GUT}}\right): h_{b}\left(M_{\mathrm{GUT}}\right): h_{\tau}\left(M_{\mathrm{GUT}}\right)=\left|\frac{1-\rho \alpha_{2} / \sqrt{3}}{\sqrt{1+\left|\alpha_{2}\right|^{2}}}\right|:\left|\frac{1-\rho \alpha_{1} / \sqrt{3}}{\sqrt{1+\left|\alpha_{1}\right|^{2}}}\right|:\left|\frac{1+\sqrt{3} \rho \alpha_{1}}{\sqrt{1+\left|\alpha_{1}\right|^{2}}}\right| .
$$

These conditions depend on two complex parameters $\alpha_{1}, \alpha_{2}$ and one real and positive parameter $\rho$. The parameters $\alpha_{1}$ and $\alpha_{2}$ describe the mixing of the components of the $S U(4)_{\mathrm{c}}$ singlet and 15-plet Higgs bidoublets, while $\rho$ is the ratio of their respective Yukawa coupling constants to the fermions of the third family. Note that monoparametric versions of the YQUCs considered within CMSSM arising by taking $\alpha_{1}=-\alpha_{2}$ for $\mu>0$ [10] or $\alpha_{1}=\alpha_{2}$ for $\mu<0$ [11] are by now experimentally excluded [9,11]. In this talk, based on Ref. $[12,13]$, we show that the YQUCs in Eq. (ㅍ.2) can become compatible with two disconnected regions of the CMSSM parameter space belonging to the hyperbolic branch (HB) of the radiative electroweak symmetry breaking (EWSB): The $M_{1 / 2}>m_{0}$ region where the neutralino, $\tilde{\chi}$, is a pure bino and may (co)annihilate strongly enough with the lighter stau, $\tilde{\tau}_{1}[14,15]$; and the $m_{0} \gg M_{1 / 2}$ region, where $\tilde{\chi}$ acquires a sizable higgsino fraction [16-20] which enhances the $\tilde{\chi}-\tilde{\chi}$ annihilation and triggers neutralino-chargino $\left(\tilde{\chi} / \tilde{\chi}_{2}-\tilde{\chi}_{1}^{+}\right)$coannihilations.

We begin by describing the cosmo- $\&$ phenomeno- logical requirements which we consider in our investigation in Sec. ॠ. Next (Sec. [3), we exhibit the resulting restrictions on the parameters of the

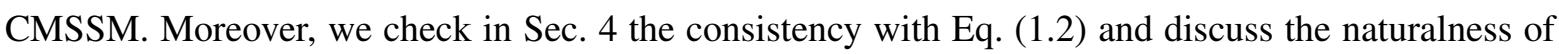
the model in Sec. [5. We summarize our conclusions in Sec. 目. 


\section{Phenomenological and Cosmological Constraints}

In our investigation, we integrate the two-loop renormalization group equations for the gauge and Yukawa coupling constants and the one-loop ones for the soft SUSY breaking parameters between $M_{\mathrm{GUT}}$ and a common SUSY threshold $M_{\mathrm{SUSY}} \simeq\left(m_{\tilde{t}_{1}} m_{\tilde{t}_{2}}\right)^{1 / 2}\left(\tilde{t}_{1,2}\right.$ are the stop mass eigenstates $)$ determined consistently with the SUSY spectrum. At $M_{\text {SUSY }}$, we impose radiative EWSB, evaluate the SUSY spectrum employing the publicly available calculator SOFTSUSY [21], and incorporate the SUSY corrections to the $b$ and $\tau$ mass - see below. From $M_{\text {SUSY }}$ to $M_{Z}$, the running of gauge and Yukawa coupling constants is continued using the standard model (SM) renormalization group equations. The SUSY spectrum is put into micrOMEGAs [22], a publicly available code which calculates a number of phenomenological - see Sec. [.] - and cosmological - see Sec. [2.2 - observables which assist us to restrict the parametric space of our model.

\subsection{Phenomenological Requirements}

2.1.1 SM Fermion Masses. After incorporating the sizable (about 20\%) and less important (almost $4 \%)$ corrections [23] to the $b$-quark and $\tau$-lepton masses, we compare the masses of top-quark, $m_{t}$, $b$-quark, $m_{b}$ and $\tau$-lepton, $m_{\tau}$ with their experimental values $[24,25]$

$$
m_{t}\left(m_{t}\right)=164.83 \mathrm{GeV}, m_{b}\left(m_{b}\right)^{\overline{\mathrm{MS}}}=4.18 \mathrm{GeV}, m_{\tau}\left(M_{Z}\right)=1.748 \mathrm{GeV} .
$$

The second value is evolved up to $M_{Z}$ using the central value $a_{s}\left(M_{Z}\right)=0.1185$ [24] of the strong fine-structure constant at $M_{Z}$ and then converted to the $\overline{\mathrm{DR}}$ scheme with result $m_{b}\left(M_{Z}\right)=2.83 \mathrm{GeV}$.

2.1.2 Collider Bounds. For our analysis, the relevant collider bounds constrain:

- The mass $m_{h}$ of the lightest Higgs boson, $h$. The experiments ATLAS [26] and CMS [27] in the LHC discovered simultaneously a boson that looks very much like the expected SM Higgs boson. The allowed $95 \%$ confidence level (c.l.) range of $m_{h}$ can be estimated including a theoretical uncertainty of about $\pm 1.5 \mathrm{GeV}$. This gives

$$
122 \lesssim m_{h} / \mathrm{GeV} \lesssim 128.5
$$

- The masses of the lightest chargino, $m_{\tilde{\chi}^{ \pm}},[28]$ and gluino, $m_{\tilde{g}}[29]$ :

$$
\text { (a) } m_{\tilde{\chi}^{ \pm}} \gtrsim 103.5 \mathrm{GeV} \text { and (b) } m_{\tilde{g}} \gtrsim 1.3 \mathrm{TeV} \text {. }
$$

2.1.3 $B$-Physics Constraints. SUSY contributions to observables related to $B$-meson physics yield restrictions to the SUSY parameters. In particular, we impose the following bounds on:

- The branching ratio $\mathrm{BR}\left(B_{s} \rightarrow \mu^{+} \mu^{-}\right)$of the process $B_{s} \rightarrow \mu^{+} \mu^{-}[30,31]$

$$
\mathrm{BR}\left(B_{s} \rightarrow \mu^{+} \mu^{-}\right) \lesssim 4.2 \times 10^{-9} .
$$

- The branching ratio $\mathrm{BR}(b \rightarrow s \gamma)$ of $b \rightarrow s \gamma[32,33]$ :

$$
2.84 \times 10^{-4} \lesssim \mathrm{BR}(b \rightarrow s \gamma) \lesssim 4.2 \times 10^{-4} .
$$

- The ratio $\mathrm{R}\left(B_{u} \rightarrow \tau \nu\right)$ of the CMSSM to the SM branching ratio of the process $B_{u} \rightarrow \tau v$ [32]

$$
0.52 \lesssim \mathrm{R}\left(B_{u} \rightarrow \tau v\right) \lesssim 2.04
$$


2.1.4 Muon Anomalous Magnetic Moment. There is a 2.9 $-\sigma$ [34-36] discrepancy

$$
\delta a_{\mu}=(24.9 \pm 8.7) \times 10^{-10} \Rightarrow 7.5 \times 10^{-10} \lesssim \delta a_{\mu} \lesssim 42.3 \times 10^{-10} \text { at } 95 \% \text { c.l. }
$$

between the measured value of the muon anomalous magnetic moment $a_{\mu}$ from its SM prediction. This $\delta a_{\mu}$ can be attributed to SUSY contributions which have the sign of $\mu$ and its absolute value decreases as $m_{\mathrm{LSP}}$ increases. Therefore, Eq. (․․) hints that the sign of $\mu$ has to be positive. Moreover, a lower [upper] bound on $m_{\text {LSP }}$ can be derived from the upper [lower] bound in Eq. (2.7). As it turns out, only the upper bound on $m_{\text {LSP }}$ is relevant here. Taking into account the aforementioned computational instabilities and the fact that a discrepancy at the level of about $3-\sigma$ cannot firmly establish a real deviation from the SM value, we restrict ourselves to just mentioning at which level Eq. (2.]) is satisfied in the parameter space allowed by all the other constraints.

\subsection{Cold Dark Matter Considerations}

2.2.1 CDM Abundance. In the context of the CMSSM, $\tilde{\chi}$ can be the lightest SUSY particle (LSP) and, thus, naturally arises as a Cold Dark Matter (CDM) candidate as long as its relic abundance does not exceed the upper bound on the CDM abundance deduced from the Planck satellite [37]

$$
\Omega_{\mathrm{LSP}} h^{2} \lesssim 0.125 \text {. }
$$

Two important mechanisms which assist to achieve $\Omega_{\mathrm{LSP}} h^{2}$ consistent with the limit above within CMSSM are (i) the coannihilation of $\tilde{\chi}$ with a particle $P$ when a proximity between the mass $m_{\text {LSP }}$ of $\tilde{\chi}$ and the mass $m_{P}$ of $P$ is established; (ii) the $P^{\prime}$-pole effect which enhances the $\tilde{\chi}-\tilde{\chi}$ pair annihilation procedure by an $P^{\prime}$-pole exchange in the $s$-channel when the mass of $P^{\prime}$ satisfies the relation $m_{P^{\prime}} \simeq$ $2 m_{\text {LSP. }}$. The strength of the coannihilation and the $P^{\prime}$-pole effect processes is controlled by the relative mass splittings. The relevant for our cases mass splittings are defined as follows

$$
\Delta_{P}=\frac{\left(m_{P}-m_{\mathrm{LSP}}\right)}{m_{\mathrm{LSP}}} \text { for } P=\tilde{\tau}_{1}, \tilde{\chi}_{1}^{+}, \tilde{\chi}_{2} \text { and } \Delta_{P^{\prime}}=\frac{m_{P^{\prime}}-2 m_{\mathrm{LSP}}}{2 m_{\mathrm{LSP}}} \text { for } P^{\prime}=H
$$

where $\tilde{\tau}_{1}$ is the lightest stau, $\tilde{\chi}_{2}$ the next-to-lightest neutralino, $\tilde{\chi}_{1}^{+}$the lightest chargino and $H$ the heavier CP-even neutral Higgs boson. The resulting $\Omega_{\mathrm{LSP}} h^{2}$ normally decreases with these $\Delta_{P}$ 's.

2.2.2 CDM Direct Detection. Employing the relevant routine of the micrOMEGAs package [38] we calculate the spin-independent (SI) and spin-dependent (SD) lightest neutralino-proton $(\tilde{\chi}-p)$ scattering cross sections $\sigma_{\tilde{\chi} p}^{\mathrm{SI}}$ and $\sigma_{\tilde{\chi} p}^{\mathrm{SD}}$, respectively. The relevant scalar, $f_{\mathrm{T}_{q}}^{p}$, and axial-vector, $\Delta_{q}^{p}$, form factors for light quarks in the proton (with $q=u, d, s$ ), are taken as follows [39,40]:

$$
\begin{aligned}
& f_{\mathrm{T} u}^{p}=0.018, f_{\mathrm{T} d}^{p}=0.026, \text { and } f_{\mathrm{T} s}^{p}=0.022 ; \\
& \Delta_{u}^{p}=+0.842, \Delta_{d}^{p}=-0.427, \text { and } \Delta_{s}^{p}=-0.085 .
\end{aligned}
$$

Data on $\sigma_{\tilde{\chi} p}^{\mathrm{SI}}$ coming from large underground Xenon (LUX) experiment [41] provide strict bounds on the values of the free parameters of SUSY models with $\tilde{\chi}$ owning a sizable higgsino component, as in our case with $m_{0} \gg M_{1 / 2}$. These data [42], however, are directly applicable in the case where the CDM consists of just $\tilde{\chi}$ 's. If the $\tilde{\chi}$ 's constitute only a part of the CDM in the universe, the LUX experiment bound on the number of the scattering events is translated into a bound on the "rescaled" SI $\tilde{\chi}-p$ elastic cross section $\xi \sigma_{\tilde{\chi} p}^{\mathrm{SI}}$, where $\xi=\Omega \tilde{\chi} / 0.12$ with 0.12 being the central value of the CDM abundance [37]. 


\section{Restrictions on the SUSY Parameters}

Imposing the requirements described in Sec. [, we can restrict the parameters of our model. Following our approach in Refs. [12,13], we consider as free parameters the ones in Eq. (페). The ratios $h_{t} / h_{\tau}$ and $h_{b} / h_{\tau}$ are then fixed by using the data of Eq. (ㅁ] $)$. These ratios must satisfy the YQUCs in Eq. (미) for natural values of the parameters $\alpha_{1}, \alpha_{2}$, and $\rho$ - see Sec. 因. To assure this, we restrict ourselves to ratios $h_{m} / h_{n}(m, n=t, b, \tau)$ close to unity which favor the range $\tan \beta \geq 40$. We also concentrate on the $\mu>0$ case, given that $\mu<0$ worsens the violation of Eq. ([2.7), and scan the region $-30 \leq A_{0} / M_{1 / 2} \leq 30$.

We localize below two separated clusters of allowed parameters categorized in the HB of the radiative EWSB, as justified in Sec. B.]: (i) The $M_{1 / 2}>m_{0}$ area at high $\tan \beta(43.8 \lesssim \tan \beta \lesssim 52)$ discussed in Sec. B.2 and (ii) the $m_{0} \gg M_{1 / 2}$ area for any $\tan \beta$ in the range $40-50$ studied in Sec. [3.3. We finally - see Sec. B.4- exhibit a direct comparison of the solutions obtained in the aforementioned areas focusing on the characteristic value $\tan \beta=48$, which balances well enough between maintaining natural values for the $h_{m} / h_{n}$ 's and satisfying the various requirements of Sec. \. Note that the numerical calculations for the soft SUSY masses become quite unstable for $m_{0} \gg M_{1 / 2}$ and $\tan \beta \gtrsim 50$.

The constraints which play an important role in delineating both allowed parameter spaces of our model are the lower bound on $m_{h}$ in Eq. (․2.) and the CDM bound in Eq. (․․). The $M_{1 / 2}>m_{0}$ region is further restricted by the bound on $\operatorname{BR}\left(B_{s} \rightarrow \mu^{+} \mu^{-}\right)$in Eq. (R.4T) whereas the $m_{0} \gg M_{1 / 2}$ area is additionally bounded by the LUX data and the limits on $m_{\tilde{\chi}^{ \pm}}$and $m_{\tilde{g}}$ in Eq. ([2.3la) and (b). In the parameter space allowed by these requirements, all the other restrictions of Sec. $\square$ are automatically satisfied - with the exception of the lower bound on $\delta a_{\mu}$ in Eq. (‥Z).

\subsection{Elliptic Versus Hyperbolic Branch}

The classification - see e.g. $[19,20]$ - of the various solutions of the radiative EWSB condition is based on the expansion of $\mu^{2}$ in terms of the soft SUSY breaking parameters of the CMSSM included in Eq. (L.]). Indeed, using fitting techniques, we can verify the following formula

$$
\mu^{2}+M_{Z}^{2} / 2 \simeq c_{0} m_{0}^{2}+c_{1 / 2} M_{1 / 2}^{2}+c_{A} A_{0}^{2}+c_{A M} A_{0} M_{1 / 2},
$$

where the coefficients $c_{0}, c_{1 / 2}, c_{A}$, and $c_{A M}$ depend basically on $\tan \beta$ and the masses of the fermions of the third generation. These coefficients are computed at the scale $M_{\text {SUSY }}-$ see Sec. $\square$ and, therefore, inherit a mild dependence on the SUSY spectrum too. From Eq. (B.]), we can easily infer that the SUSY breaking parameters are bounded above for fixed $\mu$, when the quadratic form in the right-hand side of this equation is positive definite. This is the so-called ellipsoidal branch (EB) which is highly depleted [20] after the discovery of $h$ with $m_{h}$ in the range of Eq. (2.2). On the other hand, in the HB region favored by Eq. (2.2),$c_{0}$ is negative and, consequently, $m_{0}$ can become very large together with a combination of $A_{0}$ and $M_{1 / 2}$ with all the other parameters being fixed. In this case the soft parameters lie on focal curves or surfaces. Near the boundary between the EB and HB regions, $c_{0}$ is very close to zero and, thus, $m_{0}$ can become very large with all the other parameters fixed. These are the socalled focal points. Moreover, there is a region where the soft SUSY breaking mass-squared $m_{\mathrm{H}_{2}}^{2}$ of $\mathrm{H}_{2}$ becomes independent of the asymptotic value of the parameter $m_{0}$. This is called the focus point (FP) region [18]. In the large $\tan \beta$ regime under consideration, we have [1] $m_{H_{2}}^{2} \simeq-\mu^{2}-M_{Z}^{2} / 2$ and so no distinction between focal and focus points can be established. 


\begin{tabular}{|c|cccc|cc|}
\hline REGION & $c_{0}$ & $c_{1 / 2}$ & $c_{A}$ & $c_{A M}$ & $C_{0} / 10^{8}$ & $C_{A} / 10^{7}$ \\
\hline$M_{1 / 2}>m_{0}$ & -0.059 & 0.955 & 0.102 & -0.277 & -1.05 & 6.14 \\
$m_{0} \gg M_{1 / 2}$ & -0.0689 & 0.848 & 0.0982 & -0.254 & 33.3 & -2.34 \\
\hline
\end{tabular}

Table 1: The $c$ 's in Eq. (3.1) and C's in Eq. (3.2) for $\tan \beta=48$ and $M_{1 / 2}>m_{0}$ or $m_{0} \gg M_{1 / 2}$.

To get an idea of how our solutions presented in Secs. B.2-3.4 are classified into these categories,

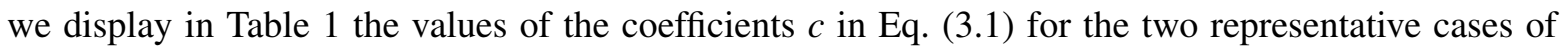
Table $\square$ corresponding to $\tan \beta=48-$ see Sec. B.4. We obtain $c_{0}<0$ in both cases and so we expect that our solutions belong to the HB region. To illustrate the emergence of the relevant focal curves we diagonalize the quadratic form in the right-hand side of Eq. (B.D) keeping, e.g., $M_{1 / 2}$ fixed and using $m_{0}$ and $A_{0}$ as varying parameters. Then, Eq. (B. $($ ) can be cast in the following form

$$
m_{0}^{2} / C_{0}+\bar{A}_{0}^{2} / C_{A}=1
$$

where $\bar{A}_{0}=A+c_{A M} M_{1 / 2} / 2 c_{A}, C_{0}=\bar{\mu}^{2} / c_{0}$, and $C_{A}=\bar{\mu}^{2} / c_{A}$ with $\bar{\mu}^{2}=\mu^{2}+M_{Z}^{2} / 2-\bar{c}_{1 / 2} M_{1 / 2}^{2}$ and $\bar{c}_{1 / 2}=c_{1 / 2}-c_{A M}^{2} / 4 c_{A}$. The numerical values of $C_{0}$ and $C_{A}$ also listed in Table $\mathbb{C}$. Since in both cases, one from $C_{0}$ and $C_{A}$ is negative, $m_{0}$ and $A_{0}$ can vary along an hyperbola parameterized by Eq. (B.2) and so both $\left(M_{1 / 2}>m_{0}\right.$ and $\left.m_{0} \gg M_{1 / 2}\right)$ our solutions in Table $\square$ belong to the HB region.

\section{2 $M_{1 / 2}>m_{0}$ Region}

Initially, we concentrate on the $M_{1 / 2}>m_{0}$ region and delineate in the left plot of Fig. $\square$ the allowed (shaded) areas in the $M_{1 / 2}-m_{0}$ plane for $\tan \beta=48$ and various $A_{0} / M_{1 / 2}$ 's indicated therein. The lower boundaries of these areas corresponds to $\Delta_{\tilde{\tau}_{1}}=0$; the areas below these boundaries are excluded because the LSP is the charged $\tilde{\tau}_{1}$. The upper boundaries of the areas come from the CDM bound in Eq. (2.8), while the left one originates from the limit on $\mathrm{BR}\left(B_{s} \rightarrow \mu^{+} \mu^{-}\right)$in Eq. (2.4). The upper right corners of the areas coincide with the intersections of the lines $\Delta_{\tilde{\tau}_{1}}=0$ and $\Omega_{\mathrm{LSP}} h^{2}=0.125$. We observe that the allowed area, starting from being just a point at a value of $A_{0} / M_{1 / 2}$ slightly bigger than -0.9 , gradually expands as $A_{0} / M_{1 / 2}$ decreases and reaches its maximal size around $A_{0} / M_{1 / 2}=-1.6$. For smaller $A_{0} / M_{1 / 2}$ 's, it shrinks very quickly and disappears just after $A_{0} / M_{1 / 2}=-1.62$. The fact that the allowed regions are narrow strips along the lines with $\Delta_{\tilde{\tau}_{1}}=0$ indicates that the main mechanism which reduces $\Omega_{\mathrm{LSP}} h^{2}$ below 0.125 is the $\tilde{\chi}-\tilde{\tau}_{1}$ coannihilations. Namely, the dominant processes are the $\tilde{\tau}_{2} \tilde{\tau}_{2}^{*}$ coannihilations to $b \bar{b}$ and $\tau \bar{\tau}$ which are enhanced by the $s$-channel exchange of $H$, with $\Delta_{H} \simeq 1.1-$ see also Table 】.

Extending our analysis to various $\tan \beta$ 's for $\Delta_{\tilde{\tau}_{1}}=0$ we can obtain a more spherical view of the overall allowed region of the model for $M_{1 / 2}>m_{0}$. This is because $\Delta_{\tilde{\tau}_{1}}=0$ ensures the maximal possible reduction of $\Omega_{\mathrm{LSP}} h^{2}$ due to the $\tilde{\chi}-\tilde{\tau}_{1}$ coannihilation and so we find the maximal $M_{1 / 2}$ or $m_{\text {LSP }}$ allowed by Eq. (2.8) for a given value of $A_{0} / M_{1 / 2}$. The relevant allowed (hatched) regions in the $M_{1 / 2}-A_{0} / M_{1 / 2}$ plane are displayed in the right plot of Fig. $\amalg$. The right boundaries of the allowed regions correspond to $\Omega_{\mathrm{LSP}} h^{2}=0.125$, while the left ones saturate the bound on $\operatorname{BR}\left(B_{s} \rightarrow \mu^{+} \mu^{-}\right)$in Eq. ([2.4). The almost horizontal upper boundaries correspond to the sudden shrinking of the allowed areas which is due to the weakening of the $H$-pole effect as $A_{0} / M_{1 / 2}$ drops below a certain value for each $\tan \beta$. The lower left boundary of the areas for $\tan \beta=44,45$, and 46 comes for the lower 

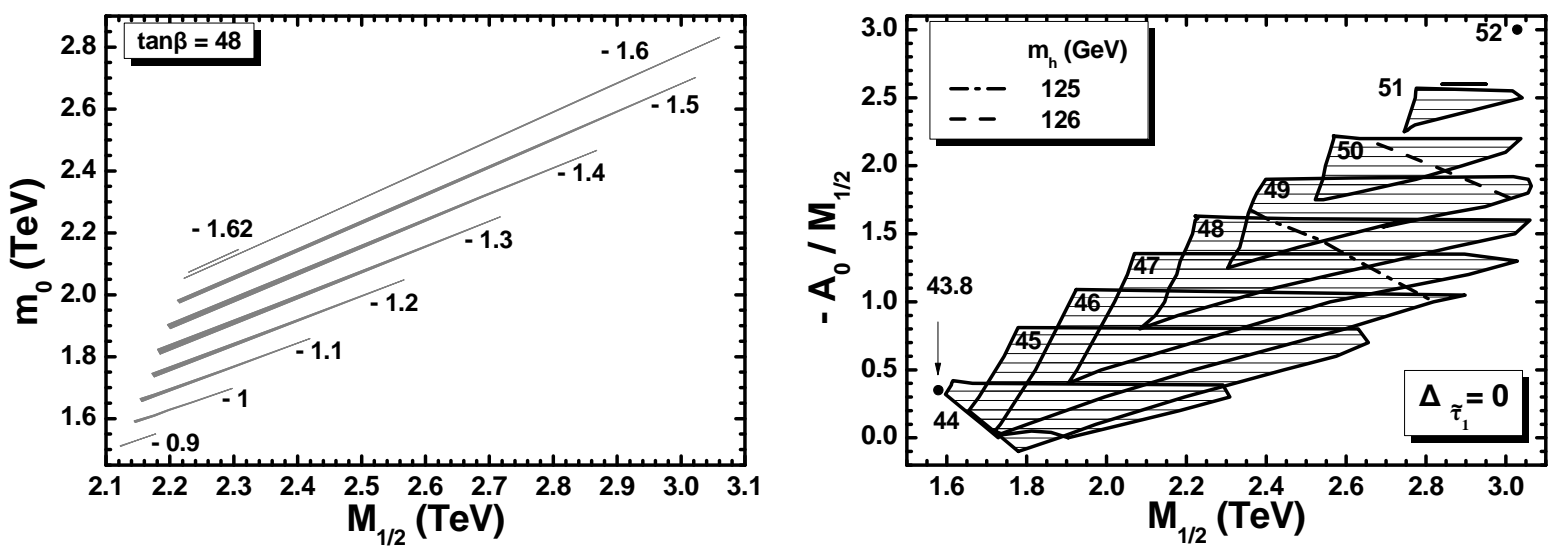

Figure 1: Allowed (shaded) areas in the $M_{1 / 2}-m_{0}\left[M_{1 / 2}-A_{0} / M_{1 / 2}\right]$ plane (left [right] plot) for $M_{1 / 2}>m_{0}$. In the left [right] plot we use $\tan \beta=48\left[\Delta_{\tilde{\tau}_{1}}=0\right]$ and various $A_{0} / M_{1 / 2}$ 's [ $\tan \beta$ 's] indicated in the graph. The dot-dashed [dashed] line corresponds to $m_{h}=125[126] \mathrm{GeV}$.

bound on $m_{h}$ in Eq. ([2.2), while the somewhat curved, almost horizontal, part of the lower boundary of the area for $\tan \beta=44$ originates from Eq. (2.8)). The dot-dashed and dashed lines correspond to $m_{h}=125$ and $126 \mathrm{GeV}$ respectively. We see that the $m_{h}$ 's which are favored by LHC can be readily obtained for $47 \lesssim \tan \beta \lesssim 50$. In the overall allowed region we obtain $122 \lesssim m_{h} / \mathrm{GeV} \lesssim 127.23$ and $746.5 \lesssim m_{\mathrm{LSP}} / \mathrm{GeV} \lesssim 1433$. Also $\delta a_{\mu} \simeq(0.35-2.76) \times 10^{-10}$ and so, Eq. (2.7) is satisfied only at the level of 2.55 to $2.82-\sigma$.

\section{3 $m_{0} \gg M_{1 / 2}$ Region}

The interplay of the various requirements of Sec. $\square$ in the $m_{0} \gg M_{1 / 2}$ region can be easily understood from Fig. 凹, where we present the (shaded) strips in the $M_{1 / 2}-m_{0}$ plane allowed by Eqs. (2.2) - (2.8) for $\tan \beta=48$ and several $A_{0} / M_{1 / 2}$ 's indicated in the graph - note that no restrictions from LUX data are applied to this plot. The upper [lower] boundary along each of these allowed strips arises from the limit on $m_{\tilde{\chi}^{ \pm}}\left[\Omega_{\mathrm{LSP}} h^{2}\right]$ in Eq. ([2.3la) [Eq. (2.8)]. On the other hand, the lower limit on $m_{h}$ in Eq. (2.2) causes the termination of the strips at low values of $m_{0}$ and $M_{1 / 2}$, whereas their termination at high values of $m_{0}$ is put in by hand in order to avoid shifting the SUSY masses to very large values. The solid lines indicate solutions with $m_{h}=125.5 \mathrm{GeV}$ - see Eq. (2.2). From this figure, we easily see the main features of the $m_{0} \gg M_{1 / 2}$ region: $m_{0}$ spans a huge range $(4-15) \mathrm{TeV}$, whereas $M_{1 / 2}[\mu]$ remains relatively low $(1-6) \mathrm{TeV}[(0.1-1) \mathrm{TeV}]$. We observe also that as $A_{0} / M_{1 / 2}$ increases from -2 to 2 the allowed strip moves to larger $M_{1 / 2}$ 's and becomes less steep.

Varying continuously $A_{0} / M_{1 / 2}$ for $\tan \beta=48, m_{h}=125.5 \mathrm{GeV}$ and taking into account the LUX data, we depict in the right plot of Fig. $\square$ the overall allowed region of the model for $m_{0} \gg M_{1 / 2}$ in the $m_{\mathrm{LSP}}-A_{0} / M_{1 / 2}$ plane. On the solid and dashed line, the bounds on $m_{\tilde{\chi}^{ \pm}}$in Eq. ([2.3la) and on $\Omega_{\mathrm{LSP}} h^{2}$ in Eq. ([2.8) are saturated, whereas the restriction from the LUX data on $\xi \sigma_{\tilde{\chi} p}^{\mathrm{SI}}$ yields the dotted boundary line. Finally, the double-dot dashed boundary lines from the limit on $m_{\tilde{g}}$ in Eq. ( $(2.3 \mathrm{~b}$ ) provide the maximal and minimal $A_{0} / M_{1 / 2}$ 's. Note that the allowed regions are almost symmetric about $A_{0} / M_{1 / 2} \simeq 2.5$. Also, we remark that $\mu$ remains almost constant $\simeq 100 \pm 20 \mathrm{GeV}$ on the solid lines 

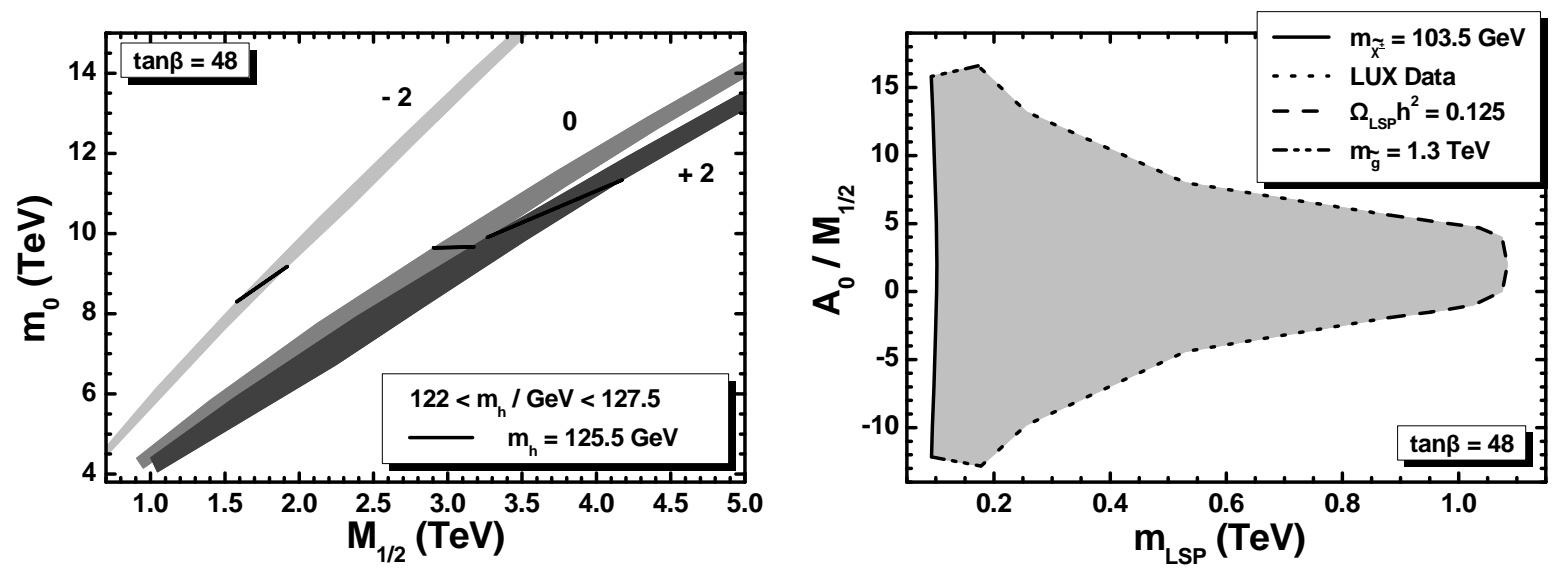

Figure 2: Allowed (shaded) areas in the $M_{1 / 2}-m_{0}\left[m_{\mathrm{LSP}}-A_{0} / M_{1 / 2}\right]$ plane (left [right] plot) for $\tan \beta=48$ and $m_{0} \gg M_{1 / 2}$. In the left plot we take various $A_{0} / M_{1 / 2}$ 's indicated in the graph and the black lines correspond to $m_{h}=125.5 \mathrm{GeV}$. In the right plot we set $m_{h}=125.5 \mathrm{GeV}$.

from Eq. ([2.3]), while it reaches about $1 \mathrm{TeV}$ when the bound in Eq. ([2.8) is saturated. Close to the latter portion of the parameter space $\Omega_{\mathrm{LSP}} h^{2}$ calculation is dominated by the $\tilde{\chi} / \tilde{\chi}_{2}-\tilde{\chi}_{1}^{+}$coannihilation processes whereas in the region where $m_{\tilde{\chi}^{ \pm}}$is near its lower limit in Eq. ( $(2.3 \mathrm{l}$ a), the $\tilde{\chi}-\tilde{\chi}$ annihilation processes contribute more efficiently to the resulting $\Omega_{\mathrm{LSP}} h^{2}$. All in all, we obtain $-12.8 \lesssim A_{0} / M_{1 / 2} \lesssim$ 15.8 and $92 \lesssim m_{\mathrm{LSP}} / \mathrm{GeV} \lesssim 1084.2$. In this area $\delta a_{\mu}$ is well below the lower limit in Eq. ([2.]), i.e. $\delta a_{\mu} \simeq(0.04-0.27) \times 10^{-10}$. Therefore, Eq. (ㅁ. $)$ is satisfied only at the level of 2.83 to $2.86-\sigma$.

In the $m_{0} \gg M_{1 / 2}$ region, where $\tilde{\chi}$ has a significant higgsino component - see Table $\square-, \sigma_{\tilde{\chi} p}^{\mathrm{SI}}$ is dominated by the $t$-channel Higgs-boson-exchange diagram contributing to the neutralino-quark elastic scattering process - for the relevant tree-level interaction terms see e.g. the appendix of Ref. [6]. Especially for large $\tan \beta$ 's, which is the case here, the couplings of $H$ to down-type quarks are proportional to $\tan \beta$ and so are the dominant ones. More explicitly, $\sigma_{\tilde{\chi} p}^{\mathrm{SI}}$ behaves as

$$
\sigma_{\tilde{\chi} p}^{\mathrm{SI}} \propto \tan ^{2} \beta\left|N_{1,1}\right|^{2}\left|N_{1,3}\right|^{2} / m_{H}^{4}
$$

where $N_{1,1}, N_{1,2}$, and $N_{1,(3,4)}$ are the elements of the matrix $N$ which diagonalizes the neutralino mass matrix and express the bino, wino, and higgsino component of $\tilde{\chi}$, respectively. As a consequence, $\sigma_{\tilde{\chi} p}^{\mathrm{SI}}$ can be rather enhanced compared to its value in the $M_{1 / 2}>m_{0}$ region, where $\tilde{\chi}$ is a pure bino.

This conclusion can be clearly induced by Fig. [3, where we show the allowed (shaded) regions in the $m_{\mathrm{LSP}}-\xi \sigma_{\tilde{\chi} p}^{\mathrm{SI}}$ plane for $\tan \beta=48$. The left [right] panel corresponds to $A_{0} / M_{1 / 2} \leq 0\left[A_{0} / M_{1 / 2} \geq 0\right]$. The numbers on the various points of each boundary line indicate the corresponding values of $A_{0} / M_{1 / 2}$. The solid, double dot-dashed, and dashed lines correspond to the bounds from Eqs. ( $2.3 \mathrm{l} a),(2.3 \mathrm{~b})$, and (2.8), respectively - cf. right plot in Fig. $\square$. The dotted lines arise from the LUX data, whereas the dot-dashed lines give the lowest possible $\xi \sigma_{\tilde{\chi} p}^{\text {SI }}$ in each case, with $\xi$ ranging from from about 0.013 to 1 (along the dashed line). From these graphs, we infer that, the maximal [minimal] $\xi \sigma_{\tilde{\chi} p}^{\mathrm{SI}}$ is located in the upper right [lowest left] corner of the allowed regions, at the junction point of the dashed and dotted [dot-dashed and solid] lines. The overall minimum of $\xi \sigma_{\tilde{\chi} p}^{\text {SI }}$ is obtained in the right plot of Fig. [] 

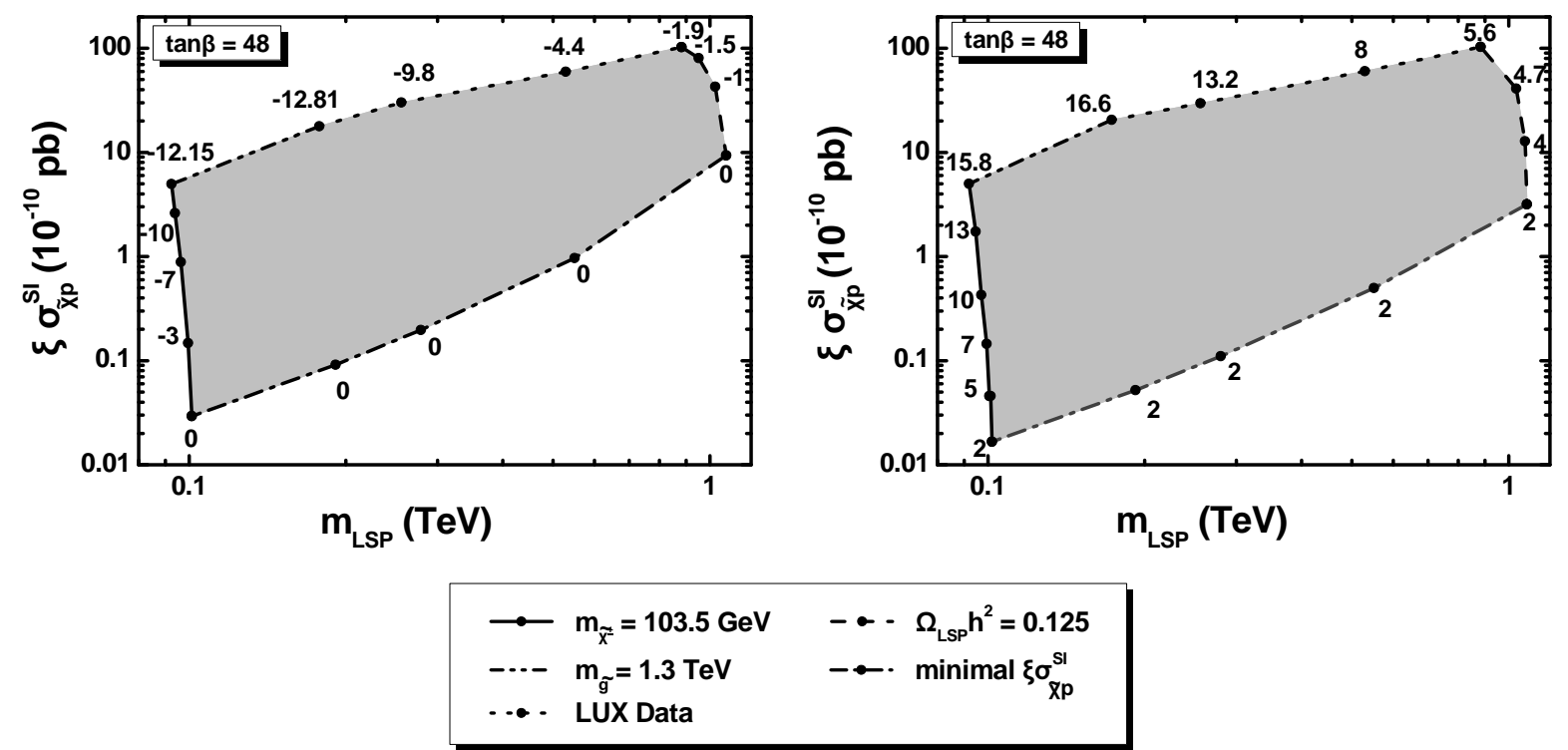

Figure 3: Allowed (shaded) regions in the $m_{\mathrm{LSP}}-\xi \sigma_{\tilde{\chi} p}^{\mathrm{SI}}$ plane for $m_{0} \gg M_{1 / 2}, m_{h}=125.5 \mathrm{GeV}$ and $\tan \beta=48$. The left [right] panel corresponds to $A_{0} / M_{1 / 2} \leq 0\left[A_{0} / M_{1 / 2} \geq 0\right]$ and the values of $A_{0} / M_{1 / 2}$ at the various points of the boundary lines are indicated. The conventions adopted for the various lines are also shown.

whereas the maximal one is practically the same in both plots. Namely we obtain

$$
1.66 \times 10^{-12} \lesssim \xi \sigma_{\tilde{\chi} p}^{\mathrm{SI}} / \mathrm{pb} \lesssim 1.03 \times 10^{-8} .
$$

As a consequence, the obtained values of $\xi \sigma_{\tilde{\chi} p}^{\text {SI }}$ are within the reach of the forthcoming experiments like XENON1T [43] and superCDMS [44].

Note, finally, that similar results, as regards the allowed ranges of $m_{\mathrm{LSP}}$ and $A_{0} / M_{1 / 2}$ and the predicted $\xi \sigma_{\tilde{\chi} p}^{\text {SI }}$ can be obtained [13] for other $\tan \beta$ 's in the range $40-50$ too.

\section{4 $M_{1 / 2}>m_{0}$ Versus $m_{0} \gg M_{1 / 2}$ Region}

Comparing the right panels of Figs. $\square$ and \ we notice that the allowed areas in the $M_{1 / 2}>m_{0}$ and $m_{0} \gg M_{1 / 2}$ regions share common $A_{0} / M_{1 / 2}$ 's for $\tan \beta=48$. Focusing on a such $A_{0} / M_{1 / 2}$ value and selecting the remaining input parameters so that we achieve the central values for $m_{h}$ and $\Omega_{\mathrm{LSP}} h^{2}$, we can implement a more direct comparison between the $M_{1 / 2}>m_{0}$ and $m_{0} \gg M_{1 / 2}$ solutions found in our work. To this end, we arrange in Table $\square$ the values of the input and some output parameters, the mass spectra and some low energy observables of the model for two characteristic points of the allowed parameter space with $\tan \beta=48, A_{0} / M_{1 / 2}=-1.5$ resulting to $\Omega_{\mathrm{LSP}} h^{2}=0.12$ and $m_{h}=125.5 \mathrm{GeV}$. The various masses of the SUSY particles (gauginos/higgsinos $\tilde{\chi}, \tilde{\chi}_{2}, \tilde{\chi}_{3}, \tilde{\chi}_{4}, \tilde{\chi}_{1}^{ \pm}, \tilde{\chi}_{2}^{ \pm}, \tilde{g}$, squarks $\tilde{t}_{1}, \tilde{t}_{2}$, $\tilde{b}_{1}, \tilde{b}_{2}, \tilde{u}_{L}, \tilde{u}_{R}, \tilde{d}_{L}, \tilde{d}_{R}$, and sleptons $\left.\tilde{\tau}_{1}, \tilde{\tau}_{2}, \tilde{v}_{\tau}, \tilde{e}_{L}, \tilde{e}_{R}\right)$ and the Higgs particles $\left(h, H, H^{ \pm}, A\right)$ are given in $\mathrm{TeV}$ - note that we consider the first two generations of squarks and sleptons as degenerate. From the values of the various observable quantities, we can verify that all the relevant constraints, but the one of Eq. (2.7), are met - cf. Sec. \. For the interpretation of our results, mainly on $\Omega_{\mathrm{LSP}} h^{2}$, we also list the values of the various $\Delta_{P}$ 's in Eq. (2.9), the bino, $\left|N_{1,1}\right|^{2}$, and the higgsino, $\left|N_{1,3}\right|^{2}+\left|N_{1,4}\right|^{2}$, purity of $\tilde{\chi}$. We also include an estimate for the EWSB fine-tuning parameter $\Delta_{\mathrm{EW}}-$ see Sec. [1. 


\begin{tabular}{|c|c|c|}
\hline \multicolumn{3}{|c|}{ INPUT PARAMETERS } \\
\hline $\tan \beta$ & \multicolumn{2}{|c|}{48} \\
\hline$-A_{0} / M_{1 / 2}$ & \multicolumn{2}{|c|}{1.5} \\
\hline$M_{1 / 2} / \mathrm{TeV}$ & 2.821 & 2.157 \\
\hline$m_{0} / \mathrm{TeV}$ & 2.522 & 9.219 \\
\hline \multicolumn{3}{|c|}{ OUTPUT PARAMETERS } \\
\hline$h_{t} / h_{\tau}\left(M_{\mathrm{GUT}}\right)$ & 1.117 & 1.107 \\
\hline$h_{b} / h_{\tau}\left(M_{\mathrm{GUT}}\right)$ & 0.623 & 0.763 \\
\hline$h_{t} / h_{b}\left(M_{\mathrm{GUT}}\right)$ & 1.792 & 1.45 \\
\hline$\mu / \mathrm{TeV}$ & 3.514 & 0.936 \\
\hline$\Delta_{\mathrm{EW}}$ & 2972 & 216.2 \\
\hline$\overline{\Delta_{\tilde{\tau}_{1}}(\%)}$ & 0.35 & 615 \\
\hline$\Delta_{H}(\%)$ & 1.14 & 94 \\
\hline$\Delta_{\tilde{\chi}_{1}^{+}}(\%)$ & 89.96 & 1.56 \\
\hline$\Delta_{\tilde{\chi}_{2}}(\%)$ & 89.95 & 1.928 \\
\hline$\left|N_{1,1}\right|^{2}$ & 1 & 0.145 \\
\hline$\left|N_{1,3}\right|^{2}+\left|N_{1,4}\right|^{2}$ & 0 & 0.852 \\
\hline \multicolumn{3}{|c|}{ MASSES IN TeV OF SPARTICLES AND HIGGSES } \\
\hline$\tilde{\chi}, \tilde{\chi}_{2}^{0}$ & $1.305,2.478$ & $0.943,0.960$ \\
\hline$\tilde{\chi}_{3}^{0}, \tilde{\chi}_{4}^{0}$ & $3.509,3.512$ & $1.034,1.959$ \\
\hline$\tilde{\chi}_{1}^{ \pm}, \tilde{\chi}_{2}^{ \pm}$ & $2.479,3.512$ & $0.956,1.959$ \\
\hline$\tilde{g}$ & 6 & 4.936 \\
\hline$\tilde{t}_{1}, \tilde{t}_{2}$ & $3.998,4.729$ & $6.309,7.270$ \\
\hline$\tilde{b}_{1}, \tilde{b}_{2}$ & $4.692,4.772$ & $7.267,7.887$ \\
\hline$\tilde{u}_{L}, \tilde{u}_{R}$ & $5.880,5.625$ & $10.1,10.004$ \\
\hline$\tilde{d}_{L}, \tilde{d}_{R}$ & $5.880,5.592$ & $10.1,9.992$ \\
\hline$\tilde{\tau}_{1}, \tilde{\tau}_{2}$ & $1.309,2.661$ & $6.749,8.202$ \\
\hline$\tilde{e}_{L}, \tilde{e}_{R}$ & $3.162,2.744$ & $9.359,9.276$ \\
\hline$\tilde{v}_{\tau}, \tilde{v}_{e}$ & $2.656,3.160$ & $8.201,9.359$ \\
\hline$h, H$ & $0.1255,2.640$ & $0.1255,3.67$ \\
\hline$H^{ \pm}, A$ & $2.641,2.640$ & $3.671,3.67$ \\
\hline \multicolumn{3}{|c|}{ LOW ENERGY OBSERVABLES } \\
\hline $10^{4} \mathrm{BR}(b \rightarrow s \gamma)$ & 3.27 & 3.3 \\
\hline $10^{9} \mathrm{BR}\left(B_{s} \rightarrow \mu^{+} \mu^{-}\right)$ & 3.74 & 3.02 \\
\hline $\mathrm{R}\left(B_{u} \rightarrow \tau v\right)$ & 0.984 & 0.991 \\
\hline $10^{10} \delta a_{\mu}$ & 0.68 & 0.227 \\
\hline$\Omega_{\mathrm{LSP}} h^{2}$ & \multicolumn{2}{|c|}{0.12} \\
\hline$\sigma_{\tilde{\chi} p}^{\mathrm{SI}} / \mathrm{pb}$ & $3.35 \cdot 10^{-12}$ & $7.28 \cdot 10^{-9}$ \\
\hline$\sigma_{\tilde{\chi} p}^{\mathrm{SD}} / \mathrm{pb}$ & $6.67 \cdot 10^{-10}$ & $8.57 \cdot 10^{-6}$ \\
\hline
\end{tabular}

Table 2: Input/output parameters, sparticle and Higgs masses, and low energy observables for $\tan \beta=48$, $A_{0} / M_{1 / 2}=-1.5$ and $M_{1 / 2}>m_{0}$ (second column) or $m_{0} \gg M_{1 / 2}$ (third column). 
The results of the two columns of Table $\square$ reveal that the $M_{1 / 2}>m_{0}$ and $m_{0} \gg M_{1 / 2}$ solutions exhibit a number of important differences: First of all, in the $m_{0} \gg M_{1 / 2}$ region, $m_{0}$ acquires considerably larger values, while $\mu$ remains quite smaller than its value for $M_{1 / 2}>m_{0}$. As a consequence, the Higgs bosons $\left(H, H^{ \pm}\right.$, and $\left.A\right)$ acquire larger masses and the whole sparticle spectrum, with the exception of the neutralinos and charginos, becomes heavier. As a by-product, the various observables besides $\Omega_{\mathrm{LSP}} h^{2}$ acquire values closer to non SUSY ones. E.g., $\delta a_{\mu}$ is even smaller than in the $M_{1 / 2}>m_{0}$ region. Note that the latter region is tightly constrained by $\mathrm{BR}\left(B_{s} \rightarrow \mu^{+} \mu^{-}\right)$, which is well suppressed for $m_{0} \gg M_{1 / 2}$. Similar values for the ratios $h_{m} / h_{n}$ with $m, n=t, b, \tau$ are obtained in both cases with results slightly closer to unity for $m_{0} \gg M_{1 / 2}$.

\begin{tabular}{|c|c|}
\hline \multicolumn{2}{|c|}{$M_{1 / 2}>m_{0}$ REGION } \\
\hline$\tilde{\tau}_{1} \tilde{\tau}_{1}^{*} \rightarrow b \bar{b}$ & $69 \%$ \\
\hline$\tilde{\tau}_{1} \tilde{\tau}_{1}^{*} \rightarrow \tau \bar{\tau}$ & $15 \%$ \\
\hline \multicolumn{2}{|c|}{$m_{0} \gg M_{1 / 2}$ REGION } \\
\hline$\tilde{\chi} \tilde{\chi}_{1}^{+} \rightarrow u \bar{d}$ & $18 \%$ \\
\hline$\tilde{\chi}_{2} \tilde{\chi}_{1}^{+} \rightarrow u \bar{d}$ & $8 \%$ \\
\hline$\tilde{\chi} \tilde{\chi}_{1}^{+} \rightarrow t \bar{b}$ & $7 \%$ \\
\hline$\tilde{\chi} \tilde{\chi}_{1}^{+} \rightarrow v_{e} \bar{e}$ & $6 \%$ \\
\hline$\tilde{\chi} \tilde{\chi} \rightarrow W^{-} W^{+}$ & $6 \%$ \\
\hline$\tilde{\chi} \tilde{\chi} \rightarrow Z Z$ & $5 \%$ \\
\hline
\end{tabular}

Table 3: Processes which contribute to $1 / \Omega_{\mathrm{LSP}} h^{2}$ more than $5 \%$ and their relative contributions for $M_{1 / 2}>m_{0}$ and $m_{0} \gg M_{1 / 2}$.

release data on $\sigma_{\tilde{\chi} p}^{\mathrm{SI}}$ and for $m_{0} \gg M_{1 / 2}$.

\section{Deviation from Yukawa Unification}

In the overall allowed parameter space of our model $[12,13]$, we find the following ranges for the ratios $h_{m} / h_{n}$ with $m, n=t, b, \tau$ :

$$
\begin{aligned}
& 0.98 \lesssim h_{t} / h_{\tau} \lesssim 1.29,0.60 \lesssim h_{b} / h_{\tau} \lesssim 0.65, \text { and } 1.62 \lesssim h_{t} / h_{b} \lesssim 2 \text { for } M_{1 / 2}>m_{0} ; \\
& 1.00 \lesssim h_{t} / h_{\tau} \lesssim 1.50,0.75 \lesssim h_{b} / h_{\tau} \lesssim 0.79, \text { and } 1.20 \lesssim h_{t} / h_{b} \lesssim 2 \text { for } m_{0} \gg M_{1 / 2}
\end{aligned}
$$

We observe that, the required deviation from YU is not so small and turns out to be comparable to the one obtained in the monoparametric case - cf. Ref. [10]. In spite of this, the restrictions from YU are not completely lost but only somewhat weakened. Actually, our model is much closer to YU than generic models with lower $\tan \beta$ 's where the Yukawa coupling constants can differ even by orders of magnitude. Also, the deviation from YU is generated by Eq. (ㅍ.2) in a natural, systematic, controlled and well-motivated way. 

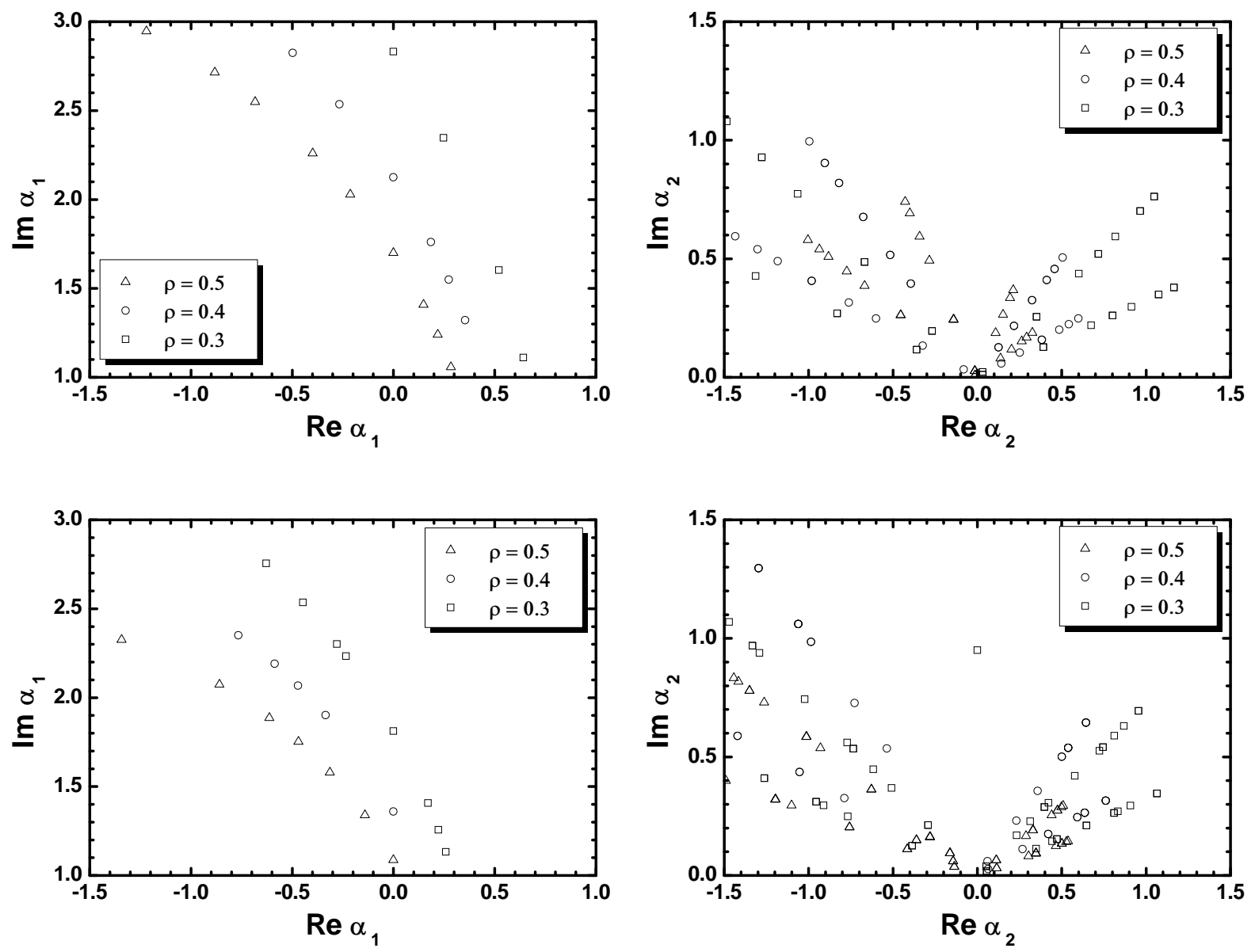

Figure 4: The complex parameters $\alpha_{1}$ and $\alpha_{2}$ for various $\rho$ 's indicated in the graphs for $\tan \beta=48, A_{0} / M_{1 / 2}=-1.5$, $m_{h}=125.5 \mathrm{GeV}$ and $M_{1 / 2}>m_{0}$ (upper plots) and $m_{0} \gg M_{1 / 2}$ (lower plots) - see Table 2 .

To show explicitly it, we below extract values of $\rho, \alpha_{1}$, and $\alpha_{2}$, which leads to the ratios $h_{m} / h_{n}$ with $m, n=t, b, \tau$, encountered in the characteristic examples presented in Table \. Since from Eq. (ㄴ.2) we have only two equations and five real unknowns we can find infinitely many solutions. Some of these solutions are shown Fig. 团 for $M_{1 / 2}>m_{0}$ (upper plots) and $m_{0} \gg M_{1 / 2}$ (lower plots) and various $\rho$ 's indicated therein. Since the equation for $h_{b} / h_{\tau}$ depends only on the combination $\rho \alpha_{1}$ its solutions, for fixed $\rho$, lie on a certain curve in the $\alpha_{1}$ complex plane, as shown in the left upper and lower panels of Fig. 因. For each $\alpha_{1}$ and $\rho$ in these panels, we find various $\alpha_{2}$ 's, depicted in the right panels of Fig. $\theta$, solving the equation for $h_{t} / h_{\tau}$. Observe that the equation for $h_{t} / h_{\tau}$ depends separately on $\alpha_{2}$ and $\rho$ and, thus, its solutions do not follow any specific pattern in the $\alpha_{2}$ complex plane. Scanning the range of $\rho$ from 0.3 to 3 and we can find solutions in the $\alpha_{1}$ and $\alpha_{2}$ planes only for the lower values of this parameter (up to about 0.6) for both allowed regions of the model. These solutions are very similar to the ones displayed in Fig. $⿴$ for all the possible values of the ratios of $h_{m} / h_{n}$ with $m, n=t, b, \tau$ allowed by the constraints of Sec. ఇ. Consequently we can safely conclude that these ratios can be readily obtained by a multitude of natural choices of the parameters $\rho, \alpha_{1}$, and $\alpha_{2}$ everywhere in the overall allowed parameter space of the model. 

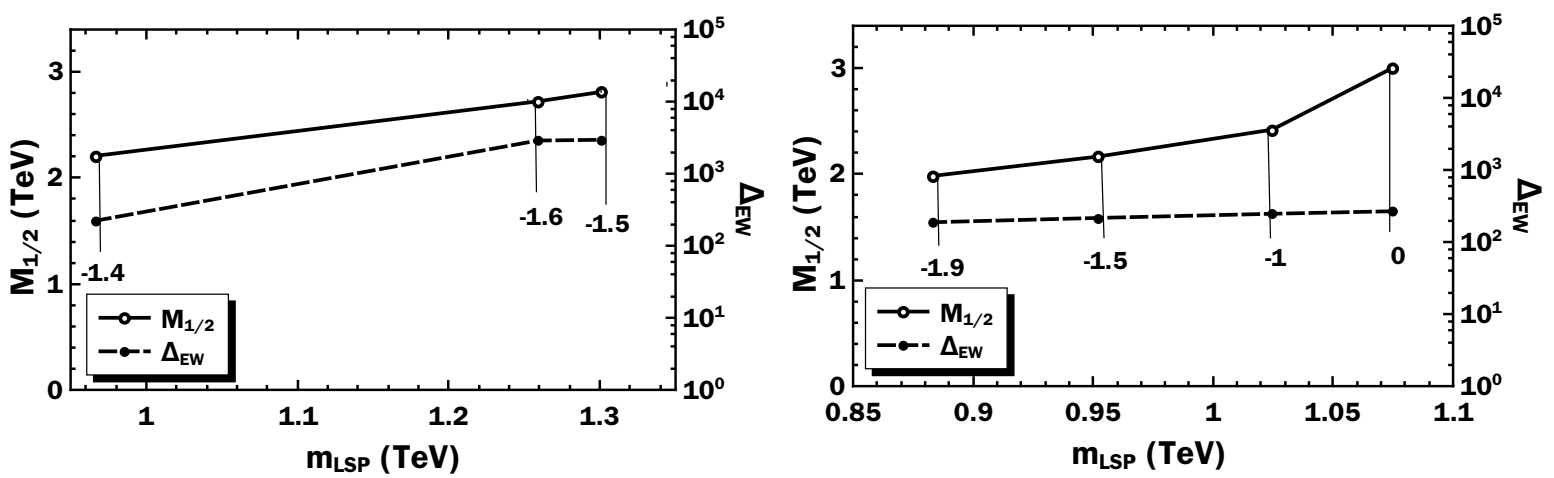

Figure 5: $M_{1 / 2}$ and $\Delta_{\mathrm{EW}}$ as functions of $m_{\mathrm{LSP}}$ for $m_{h}=125.5 \mathrm{GeV}, \Omega_{\mathrm{LSP}} h^{2}=0.125$ and various $A_{0} / M_{1 / 2}$ 's indicated on the curves for the $M_{1 / 2}>m_{0}$ (left panel) and the $m_{0} \gg M_{1 / 2}$ (right panel) region of the model.

\section{Naturalness of the EWSB}

The fact that, in our model, $M_{1 / 2}, m_{0}$, and $\mu$ generally turn out to be of the order of a few $\mathrm{TeV}$ puts under some stress the naturalness of the radiative EWSB giving rise to the so-called little hierarchy problem. To quantified somehow this issue we introduce the EWSB fine-tuning parameter

$$
\Delta_{\mathrm{EW}} \equiv \max \left(\frac{\left|C_{i}\right|}{M_{Z}^{2} / 2}\right) \quad \text { with } \quad\left(C_{\mu}, C_{H_{1}} C_{H_{2}}\right)=\left(-\mu^{2}, \frac{m_{H_{1}}^{2}}{\tan ^{2} \beta-1},-\frac{m_{H_{2}}^{2} \tan ^{2} \beta}{\tan ^{2} \beta-1}\right) .
$$

Here $i=\mu, H_{1}, H_{2}$ and $m_{H_{j}}$ is the soft SUSY breaking mass of $H_{j}$ with $j=1,2$. In most of the parameter space explored, $\Delta_{\mathrm{EW}}$ is dominated by the term $C_{\mu}$.

Focusing on the values of the parameters which ensure $\Omega_{\mathrm{LSP}} h^{2} \simeq 0.125$, we present, in the left [right] panel of Fig. [, $M_{1 / 2}$ (solid line) and $\Delta_{\mathrm{EW}}$ (dashed line) as functions of $m_{\mathrm{LSP}}$ for $M_{1 / 2}>m_{0}$ $\left[m_{0} \gg M_{1 / 2}\right], \tan \beta=48, m_{h}=125.5 \mathrm{GeV}$, and negative $A_{0} / M_{1 / 2}$ 's indicated in the graphs. The $A_{0} / M_{1 / 2}$ 's fulfilling these conditions are shown in Fig. 3 of Ref. [12] for $M_{1 / 2}>m_{0}$ and in the left panel of Fig. [ ] for $m_{0} \gg M_{1 / 2}$. We clearly see that, in both regions, the resulting $\Delta_{\text {EW }}$ is almost constant and $\Delta_{\mathrm{EW}} \sim 2000$ for $M_{1 / 2}>m_{0}$ whereas $\Delta_{\mathrm{EW}} \sim 200$ for $m_{0} \gg M_{1 / 2}$ in agreement with the values shown in Table $\square$. In other words, $\Delta_{\mathrm{EW}}$ in the $M_{1 / 2}>m_{0}$ area becomes about a factor of ten larger than its value in the $m_{0} \gg M_{1 / 2}$ area despite the fact that the resulting $m_{\mathrm{LSP}}$ 's are comparable. The crucial difference between the two regions, though, is the lower $\mu$ 's encountered for $m_{0} \gg M_{1 / 2}$ - see Table $\square-$ which largely influences the $\Delta_{\mathrm{EW}}$ computation - see Eq. (D.J). We can conclude, therefore, that the $m_{0} \gg M_{1 / 2}$ solutions are more natural regarding the EWSB fine-tuning than those for $M_{1 / 2}>m_{0}$.

\section{Conclusions}

We investigated the compatibility of the generalized asymptotic YQUCs in Eq. ([.2), which yield acceptable masses for the fermions of the third family, with the CMSSM for $\mu>0$ and $40 \leq \tan \beta \leq 50$. We imposed phenomenological constraints originating from the mass of the lightest neutral CP-even Higgs boson, the lower bounds on the masses of the sparticles, and $B$-physics. We also considered cosmological constraints coming from $\Omega_{\mathrm{LSP}} h^{2}$ and the LUX data on $\xi \sigma_{\tilde{\chi} p}^{\mathrm{SI}}$. 
We found that $\tilde{\chi}$ can act as a CDM candidate in the following two separated regions classified in the HB of the EWSB:

- The $M_{1 / 2}>m_{0}$ region, where the LSP turns out to be an essentially pure bino and $\Omega_{\mathrm{LSP}} h^{2}$ is reduced efficiently, thanks to $H$-pole enhanced stau-antistau coannihilations, so that it is compatible with the recent data on $\mathrm{BR}\left(B_{s} \rightarrow \mu^{+} \mu^{-}\right)$. The LHC preferred values $m_{h} \simeq(125-126) \mathrm{GeV}$ can be accommodated for $48 \lesssim \tan \beta \lesssim 50$, whereas $m_{\text {LSP }}$ comes out to be large $(\sim 1 \mathrm{TeV})$. As a consequence, the $\tilde{\chi}$ direct detectability is very difficult and the EWSB fine-tuning becomes rather aggressive since $\Delta_{\mathrm{EW}}{ }^{-1} \sim 0.035 \%$.

- The $m_{0} \gg M_{1 / 2}$ region, where the LSP is a bino-higgsino admixture and has an acceptable $\Omega_{\mathrm{LSP}} h^{2}$ thanks to $\tilde{\chi}-\tilde{\chi}$ annihilations (for low $m_{\mathrm{LSP}}$ 's) and the $\tilde{\chi} / \tilde{\chi}_{2}-\tilde{\chi}_{1}^{+}$coannihilations (for large $m_{\mathrm{LSP}}$ 's). Fixing $m_{h}=125.5 \mathrm{GeV}$ favored by the LHC, we found a wider allowed parameter space with $40 \lesssim \tan \beta \lesssim 50,-11 \lesssim A_{0} / M_{1 / 2} \lesssim 15,0.09 \lesssim m_{\mathrm{LSP}} / \mathrm{TeV} \lesssim 1.1$ and milder EWSB fine-tuning since $\Delta_{\mathrm{EW}}{ }^{-1} \sim 0.5 \%$. The LSP is possibly detectable in the planned CDM direct search experiments which look for $\sigma_{\tilde{\chi} p}^{\mathrm{SI}}$.

In both cases above, the restriction on $\delta a_{\mu}$ is only satisfied at a level of above $2-\sigma$ and the required deviation from YU can be easily attributed to a multitude of natural values of the relevant parameters within a PS SUSY GUT model.

\section{Acknowledgments}

This research was supported from the MEC and FEDER (EC) grants FPA2011-23596 and the Generalitat Valenciana under grant PROMETEOII/2013/017.

\section{References}

[1] V. Barger, M. Berger, and P. Ohmann, Phys. Rev. D 49, 4908 (1994).

[2] B. Ananthanarayan, G. Lazarides, and Q. Shafi, Phys. Rev. D 44, 1613 (1991).

[3] M.S. Carena, M. Olechowski, S. Pokorski, and C.E.M. Wagner, Nucl. Phys. B426, 269 (1994).

[4] M. Gómez, G. Lazarides, and C. Pallis, Nucl. Phys. B 638, 165 (2002).

[5] M.E. Gómez and C. Pallis, hep-ph/0303098.

[6] G. Lazarides and C. Pallis, hep-ph/0406081.

[7] N. Karagiannakis, G. Lazarides, and C. Pallis, Int. J. Mod. Phys. A 28, 1330048 (2013).

[8] I. Antoniadis and G. Leontaris, Phys. Lett. B 216, 333 (1989).

[9] N. Karagiannakis, G. Lazarides, and C. Pallis, J. Phys. Conf. Ser. 384, 012012 (2012).

[10] N. Karagiannakis, G. Lazarides, and C. Pallis, Phys. Lett. B 704, 43 (2011).

[11] M. Gómez, G. Lazarides, and C. Pallis, Phys. Rev. D 67, 097701 (2003).

[12] N. Karagiannakis, G. Lazarides, and C. Pallis, Phys. Rev. D 87 (2013).

[13] N. Karagiannakis, G. Lazarides, and C. Pallis, arXiv:1503.06186. 
[14] J.R. Ellis, T. Falk, and K.A. Olive, Phys. Lett. B 444, 367 (1998).

[15] M. Gómez, G. Lazarides, and C. Pallis, Phys. Rev. D 61, 123512 (2000).

[16] H. Baer, C.-h. Chen, C. Kao, and X. Tata, Phys. Rev. D 52, 1565 (1995).

[17] K. L. Chan, U. Chattopadhyay, and P. Nath, Phys. Rev. D 58, 096004 (1998).

[18] J. L. Feng, K. T. Matchev, and T. Moroi, Phys. Rev. Lett. 84, 2322 (2000).

[19] S. Akula, M. Liu, P. Nath, and G. Peim, Phys. Lett. B 709, 192 (2012).

[20] M. Liu and P. Nath, Phys. Rev. D 87, 095012 (2013).

[21] B. C. Allanach, Comput. Phys. Commun. 143, 305 (2002).

[22] G. Belanger, F. Boudjema, A. Pukhov, and A. Semenov, http://lapth.in2p3.fr/micromegas;

G. Belanger et al.. Comput. Phys. Commun. 182, 842 (2011).

[23] D. Pierce, J. Bagger, K. Matchev, and R.-J. Zhang, Nucl. Phys. B 491, 3 (1997).

[24] J. Beringer et al. (Particle Data Group), Phys. Rev. D 86, 010001 (2012).

[25] ATLAS, CDF, CMS, and D0 Collaborations, arXiv:1403.4427.

[26] G. Aad et al. (ATLAS Collaboration), Phys. Rev. D 90, 052004 (2014).

[27] CMS Collaboration, Tech. Rep. CMS-PAS-HIG-14-009 (2014).

[28] LEPSUSYWG, ALEPH, DELPHI, L3, OPAL Experiments, http://lepsusy.web.cern.ch/lepsusy.

[29] The ATLAS Collaboration, Tech. Rep. ATLAS-CONF-2013-061 (2013).

[30] R. Aaij et al. (LHCb Collaboration), Phys. Rev. Lett. 108, 231801 (2012).

[31] J. Albrecht, Mod. Phys. Lett. A 27, 1230028 (2012).

[32] E. Barberio et al. [Heavy Flavor Averaging Group], arXiv:0808.1297.

[33] M. Misiak et al., Phys. Rev. Lett. 98, 022002 (2007).

[34] M. Davier, A. Hoecker, B. Malaescu, and Z. Zhang, Eur. Phys. J. C 71, 1515 (2011).

[35] K. Hagiwara, R. Liao, A. Martin, D. Nomura, and T. Teubner, J. Phys. G 38, 085003 (2011).

[36] G. Bennett et al. (Muon g-2 Collaboration), Phys. Rev. D 73, 072003 (2006).

[37] P. Ade et al. (Planck Collaboration), Astron. Astrophys. 571, A16 (2014).

[38] G. Belanger, F. Boudjema, A. Pukhov, and A. Semenov, Comput. Phys. Commun. 180, 747 (2009)

[39] G. Belanger, F. Boudjema, A. Pukhov, and A. Semenov, Comput. Phys. Commun. 185, 960 (2014).

[40] P. Shanahan, A. Thomas, and R. Young, Phys. Rev. D 87, 074503 (2013).

[41] D. Akerib et al. (LUX Collaboration), Phys. Rev. Lett. 112, 091303 (2014).

[42] Brown University's Particle Astrophysics Group, http://dmtools . brown.edu.

[43] E. Aprile et al. (XENON1T Collaboration), Springer Proc. Phys. 148, 93 (2013), arXiv: 1206.6288.

[44] D. S. Akerib et al., Nucl. Instrum. Meth. A 559, 411 (2006).

[45] M. Aartsen et al., (IceCube Collaboration), Phys. Rev. Lett. 110, 131302 (2013). 